\title{
Programming of neural progenitors of the adult subependymal zone towards a
} glutamatergic identity by Neurogenin2

Sophie Péron ${ }^{1,2 *}$, Leo M Miyakoshi ${ }^{3,4 *}$, Monika S Brill ${ }^{3,5 *}$, Felipe Ortega ${ }^{1,2,6}$, Marisa Karow ${ }^{1,2,3}$, Sergio Gascón ${ }^{3,7,8 \$}$, Benedikt Berninger ${ }^{1,2,3 \$}$

${ }^{1}$ Research Group “Adult Neurogenesis and Cellular Reprogramming”, Institute of Physiological Chemistry, University Medical Center Johannes Gutenberg University, Mainz, Germany

${ }^{2}$ Focus Program Translational Neuroscience, Johannes Gutenberg University, Mainz, Germany

${ }^{3}$ Institute of Physiology, Department of Physiological Genomics, Biomedical Center (BMC), Ludwig-Maximilians University Munich, Germany

${ }^{4}$ Biophysics Institute Carlos Chagas Filho, Federal University of Rio de Janeiro, Rio de Janeiro, Brazil

${ }^{5}$ Institute of Neuronal Cell Biology, Technische Universität München, Munich, Germany

${ }^{6}$ Department of Biochemistry and Molecular Biology IV, Faculty of Veterinary Medicine, Complutense University of Madrid, Spain

${ }^{7}$ Institute of Stem Cell Research, Helmholtz Zentrum München, Neuherberg, Germany

${ }^{8}$ Department of Toxicology and Pharmacology, Faculty of Veterinary Medicine, Complutense University of Madrid, Spain

* Co-first author

$\$$ Co-senior author

Running title: Fate switch of NSCs-derived neurons

Keywords: adult neurogenesis, subependymal zone, Neurog2, olfactory bulb, reprogramming, proneural gene

Summary statement: Our study identifies a critical developmental time window during which progenitors of the adult subependymal zone, specified for generating GABAergic neurons, can be reprogrammed towards glutamatergic neurogenesis.

\section{Correspondence:}

Dr. Benedikt Berninger: Phone: 0049613139 21334; Email: berningb@uni-mainz.de

Dr. Sergio Gascón: Phone: 0049892180 71917; Email: sergio.gascon@med.uni-muenchen.de 


\section{ABSTRACT}

While the adult subependymal zone (SEZ) harbors pools of distinct neural stem cells that generate different types of GABAergic interneurons, a small progenitor population in the dorsal SEZ expresses Neurog2 and gives rise to glutamatergic neurons. Here we investigated whether SEZ progenitors can be programmed towards glutamatergic neurogenesis through forced expression of Neurog2. Retrovirus-mediated expression of Neurog2 induced the glutamatergic neuron lineage markers Tbr2 and Tbr1 in cultured SEZ progenitors which subsequently differentiated into functional glutamatergic neurons. Likewise, retrovirusmediated expression of Neurog2 in dividing SEZ progenitors within the adult SEZ induced Tbr2 and Tbr1 expression, hallmarking entry into the glutamatergic lineage also in vivo. Intriguingly, Neurog2-expressing progenitors failed to enter the rostral migratory stream (RMS) and instead differentiated directly within the SEZ or the adjacent striatum. In sharp contrast, lentivirus-mediated postmitotic expression of Neurog2 failed to reprogram early SEZ neurons, which instead maintained their GABAergic identity and migrated along the RMS towards the olfactory bulb. Thus, our data show that Neurog2 can program SEZ progenitors towards a glutamatergic identity, but fails to reprogram their postmitotic progeny. 


\section{INTRODUCTION}

Accruing evidence indicates that neural stem cells (NSCs) lining the walls of the lateral ventricle in the postnatal and adult subependymal zone (SEZ) exhibit regional identity thereby conferring specific fate restrictions on NSCs (Azim et al., 2015; Lim and Alvarez-Buylla, 2014). Due to this characteristic mosaic organization of the SEZ, NSCs residing in different SEZ domains along the rostro-caudal and dorsal-ventral axes generate neurons of distinct subtype identities and become subsequently destined for distinct sub-domains within the olfactory bulb (OB) (Brill et al., 2009; Merkle et al., 2014; Merkle et al., 2007; Sequerra, 2014). Grafting experiments indicate that these region-specific identities do not become erased upon placing NSCs into heterotopic locations, arguing that extrinsic signals provided locally are not sufficient to reprogram the fate restrictions of NSCs (Merkle et al., 2007). Furthermore, regional fate restrictions appear to extend even to the decision between neuronal and glial fates as indicated by the fact that oligodendrogliogenic NSCs are enriched in the dorsal SEZ and and in vitro clearly constitute a lineage distinct from neurogenic NSCs (Ortega et al., 2013).

While the majority of NSCs from the adult SEZ give rise to several types of GABAergic or tyrosine hydroxylase-expressing interneurons (Lim and Alvarez-Buylla, 2014), previous work has shown that a small subpopulation of NSCs located in the dorsal SEZ can generate juxtaglomerular glutamatergic neurons (Brill et al., 2009). This subpopulation is characterized by sequential expression of Pax6, Neurog2, Tbr2, and Tbr1 (Brill et al., 2009) that characterizes glutamatergic neuron producing lineages throughout the forebrain (Hevner et al., 2006). Forced transcription factor expression can alter fate restrictions of neural cells beyond the stem cell stage (Arlotta and Berninger, 2014). Forced expression of Pax6 (Hack et al., 2005), Dlx2 (Brill et al., 2008), and Fezf2 (Zuccotti et al., 2014) has been shown to shift subtype specification within the adult SEZ in vivo. When cultured under neurosphere-conditions (high concentration of epidermal growth factor (EGF) and fibroblast growth factor-2, (FGF2)), adult SEZ stem and progenitor cells could be directed towards generation of fully functional glutamatergic neurons by retrovirus-mediated expression of Neurog2 (Berninger et al., 2007b). Moreover, upon transplantation into the adult hippocampal dentate gyrus, Neurog2-expressing adult SEZ stem or progenitor cells exhibited morphological similarities to endogenous dentate granule neurons and some degree of functional integration (Chen et al., 2012). However, exposure to EGF and FGF2 exerts dramatic effects on NSCs that may include a partial loss of regional specification (Gabay et al., 2003; Hack et al., 2004) and may render these cells more permissive to Neurog2. Thus, in the present study we addressed the question whether Neurog2 can overcome fate restrictions of adult SEZ stem and progenitor cells in the absence of elevated growth factor signaling and drive 
83 these towards acquisition of a glutamatergic phenotype, and if so, whether such effect extends 84 into the postmitotic life of adult-generated SEZ-derived neurons.

85 


\section{RESULTS}

Forced expression of Neurog2 programs adult SEZ progenitors towards a glutamatergic identity in vitro

Given that adult-generated glutamatergic OB neurons originate from progenitors located in the dorsal part of the SEZ that express the proneural gene Neurog2 (Brill et al., 2009) we wondered whether ectopic expression of this transcription factor can program progenitors from the ventral SEZ, which otherwise give rise exclusively to GABAergic neurons, towards a glutamatergic neuron identity. To address this question we first took advantage of an adherent culture of the adult SEZ (Costa et al., 2011; Ortega et al., 2011), which preserves the ratio of GABAergic and glutamatergic neurogenesis as observed in vivo (Brill et al., 2009) and transduced proliferating progenitors with a retrovirus encoding Neurog2, followed by the reporter DsRed behind an internal ribosomal entry site (IRES; Fig. 1A). While cultures infected with a control virus contained only very few neurons (identified by MAP2) expressing the vesicular glutamate transporter-1 (vGluT1), forced expression of Neurog2 resulted in a massive up-regulation of vGluT1 (20-30 days post infection, DPI), localized to puncta presumably reflecting presynaptic terminals (Fig. 1B). To confirm an actual glutamatergic re-specification, we directly assessed whether Neurog2-expressing neurons exhibit glutamatergic synaptic transmission. To this end, we performed pair recordings of neurons derived from Neurog2-expressing progenitors and nontransduced control neurons 4 weeks after transduction (Fig. 1C). While synaptic transmission mediated by control neurons was GABAergic in nature (Fig. 1C) as described previously (Costa et al., 2011), stimulation of Neurog2-expressing cells in these pairs resulted in postsynaptic currents that were fully blocked by the 2-amino-3-(3-hydroxy-5-methyl-isoxazol-4-yl)propanoic acid (AMPA)/kainate receptor antagonist 6-cyano-7-nitroquinoxaline-2,3-dione (CNQX), demonstrating its glutamatergic nature (Fig. 1C) $(\mathrm{n}=6$ pairs analyzed). This data demonstrates that forced expression of Neurog2 can program adult SEZ progenitors towards a glutamatergic identity.

\section{Neurog2-mediated fate conversion of adult neural stem cell progeny in vivo}

Next, we investigated the effects of forced Neurog2 expression in the progeny of adult SEZ NSCs in vivo. To this end, we stereotactically injected retroviruses encoding DsRed only, for control, or Neurog2-IRES-DsRed as experimental manipulation into the adult SEZ. As expected, after seven days, control virus infection revealed the characteristic picture of retrovirally labeled cells migrating in chains along the entire extent of the rostral migratory stream (RMS) towards the core of the olfactory bulb (OB) (Lois et al., 1996). Indeed, $44 \pm 7 \%$ of cells were found in the 
SVZ, $19 \pm 10 \%$ of cells were migrating in the RMS and $37 \pm 9 \%$ of cells reached the OB ( $\mathrm{n}=3$ mice, 10197 cells analyzed, Fig. 2A-D and I). The DsRed-positive cells in the SEZ (Fig. 2B) and the RMS (Fig. 2C) exhibited morphologies of migratory neuroblasts and upon reaching the OB dispersed radially to establish themselves within the granular and glomerular layer (Fig. 2D). In sharp contrast, progenitors expressing Neurog2 exhibited a drastically altered migratory behavior $(\mathrm{n}=3$ mice, 823 cells analyzed), abandoning chain migration $(1.5 \pm 1 \%$ of cells in the RMS) (Fig. 2E,G,I). As a consequence, only extremely few cells finally reached the OB (2 $\pm 2 \%$ ) (Fig. 2E,H,I), while most of them remained in the SEZ area (96 $\pm 1 \%$ ) (Fig. 2E,F,I). Moreover, although the overall proportion of DCX-positive cells did not differ between control and Neurog2-expressing cells (Fig. 3A,B and E-G), Neurog2-expressing neurons differentiated within the SEZ or the adjacent striatum, with a highly complex dendritic arborization and high density of dendritic spines (Fig. 3C,D) characteristic of projection neurons. None of these morphologies were observed upon injection of the control virus. To further assess whether Neurog2-transduced cells acquire a molecular glutamatergic neuron identity in vivo, we stained for the T-box transcription factors Tbr2 and Tbr1, hallmarks of glutamatergic neurogenesis (Hevner et al., 2006). Remarkably, Tbr2 was found to be expressed in $36 \pm 8 \%$ of Neurog2expressing neurons ( $\mathrm{n}=3$ mice, 7 DPI, 95 cells analyzed) located in the ventral SEZ, which normally is devoid of cells expressing this transcription factor $(0.7 \pm 0.3 \%$ of Tbr2-positive cells among cells infected with the control retrovirus, $n=3$ mice, 7 DPI, 962 cells analyzed) (Fig. 3HJ). The acquisition of a glutamatergic neuron program by Neurog2-expressing cells was further corroborated by the presence of a similar proportion of Tbr1-positive cells $(n=2,7$ DPI, 215 cells analyzed) (Fig. 3K).

\section{Forced expression of Neurog2 in young postmitotic neurons does not result in} reprogramming towards a glutamatergic phenotype

In the above experiments, use of retroviral vectors encoding Neurog2 restricted transduction to fast-dividing cells, most of which are transit-amplifying precursors (Costa et al., 2011; Doetsch et al., 2002). We next asked whether conversion towards a glutamatergic identity would be possible at later stages of lineage progression, i.e., after the last cell division when these cells become postmitotic and commence differentiation.

Thus, we employed lentiviral vectors encoding Neurog2 and egfp allowing for transduction of non-dividing cells. In these constructs, expression of Neurog2 and the reporter are driven by the minimal human synapsin promoter (hSyn, Fig. 4A) restricting expression of Neurog2 and egfp to postmitotic neurons (Gascon et al., 2008). In fact, transduction of primary cortical cultures from 
embryonic day E14 with the lentiviral vector hSyn-Neurog2-IRES-egfp resulted in efficient expression of Neurog2 and GFP proteins, which were both restricted to Tuj-1-immunoreactive neurons (Fig. 4B). We then examined whether hSyn-driven expression of Neurog2 in adult SEZ cultures causes a similar conversion towards a glutamatergic identity as observed following transduction at the precursor stage. In sharp contrast to the effect of Neurog2 observed in dividing progenitors, the vast majority of the lentivirus-transduced neurons remained immunostaining-negative for vGluT1 at 30 days post infection (Fig. 4C), although vGluT1 positive neurons were readily detectable (Fig. 4D). However, their number did not differ from cultures transduced with a control lentiviral vector $(2.5 \%$ vs $4 \%, 701$ cells analyzed) (Fig. 4E), indicating that these few glutamatergic neurons represent the small endogenous glutamatergic population derived from the dorsal portion of the adult SEZ (Brill et al., 2009). This data show that forced Neurog2 expression fails to convert immature postmitotic neurons derived from adult NSCs into glutamatergic neurons in vitro.

\section{Neurog2 activates the transcriptional program of glutamatergic neurogenesis in SEZ progenitors, but fails to do so in young postmitotic neurons}

Forebrain glutamatergic neurogenesis is generally characterized by the sequential expression of Tbr2 and Tbr1 (Hevner et al., 2006) and previous work has delineated the same expression sequence in glutamatergic neurons derived from Neurog2-expressing SEZ progenitors (Brill et al., 2009). Thus, we examined whether forced Neurog2 expression results in the activation of the same molecular pathway in adult SEZ progenitor cells. To this end, we transduced cultured SEZ progenitors with the retrovirus encoding Neurog2 and analyzed Tbr2 and Tbr1 expression at days 7 and 9 post infection, respectively. Consistent with the natural program of glutamatergic neurogenesis we observed expression of both transcription factors in Neurog2-expressing cells (Fig. 5A,B). Tbr2 was expressed only in a subpopulation of Neurog2-transduced cells ( $44 \pm 3 \%$; Fig. 5D), most likely due to the fact that this transcription factor is expressed transiently during glutamatergic lineage progression (Hevner et al., 2006). We next transduced adult SEZ cultures with the LV-hSyn-Neurog2-IRES-egfp. Contrary to the effect of retroviral expression at the progenitor stage, expression of Neurog2 in immature postmitotic neurons did not induce Tbr2 (Fig. 5A,D) or Tbr1 (data not shown) expression. This was more conspicuous as Tbr2 was found to be expressed in a minor subpopulation of untransduced SEZ cells ( $\pm 5 \%$; Fig. 5A), again reflecting the low degree of intrinsic glutamatergic neurogenesis from adult SEZ progenitors (Brill et al., 2009). Finally, in agreement with the failure to activate a glutamatergic program, neurons transduced with LV-hSyn-Neurog2-IRES-egfp maintained GABA immunoreactivity (94 
$\pm 6 \%$; Fig. 5C,D), while retrovirus-mediated Neurog2 expression at the progenitor stage resulted in a loss of GABA immunoreactivity in line with the acquisition of a glutamatergic phenotype (Fig. 5C,D). Thus, this data not only confirm that Neurog2-transduced SEZ progenitors undergo a fate conversion, but that this involves the recapitulation of glutamatergic neurogenesis from adult SEZ progenitors under physiological conditions (Brill et al., 2009). On the other hand, the failure of inducing a glutamatergic phenotype in young postmitotic neurons following forced Neurog2 expression is also accompanied by failure of inducing the stereotypical program of glutamatergic lineage progression.

\section{Neurog2 expression in young postmitotic neurons fails to induce a glutamatergic phenotype in vivo}

We next aimed to assess the effect of forced Neurog2 expression in young postmitotic neurons following stereotactic injection of LV-hSyn-Neurog2-IRES-egfp into the adult SEZ in vivo (Fig. 6). In contrast to the aberrant migration of cells transduced with a retrovirus encoding Neurog2 (Fig. 2), hSyn-driven Neurog2 expression did not result in alterations in the migration program. Indeed, DCX-positive cells were found to migrate normally via the RMS towards the OB (control: $9 \pm 4.5 \%$ of cells in SEZ, $3 \pm 0.6 \%$ of cells in RMS and $88 \pm 5 \%$ of cells in OB, $n=3$ mice, 1929 cells analyzed; Neurog2: $10 \pm 4 \%$ of cells in SEZ, $2.5 \pm 1 \%$ of cells in RMS and $88 \pm$ $5.5 \%$ of cells in OB, $n=3$ mice, 855 cells analyzed; Fig. 6A). The presence of DCX-positive cells expressing GFP while leaving the RMS indicates the early activity of the hSyn-promoter in SEZ cells transduced with the LV-hSyn-Neurog2-IRES-egfp (Fig. 6B). Notably, at 10 DPI a large number of transduced cells were already present in the $\mathrm{OB}$ and dispersed radially towards the more superficial layers, where the majority acquired the morphology characteristic of GABAergic granule or periglomerular neurons (Fig. 6C,D). Also at that late stage, we were unable to detect expression of the glutamatergic lineage marker Tbr2 in both, granule or periglomerular neurons, amongst the LV-hSyn-Neurog2-IRES-egfp cells (Fig. 6E-F). This data indicate that Neurog2 expression at a postmitotic stage fails to reprogram the phenotype of neurons derived from adult NSCs. 


\section{DISCUSSION}

In the present study we demonstrate that forced expression of Neurog2 redirects the program of proliferating adult SEZ progenitors, mostly giving rise to GABAergic olfactory neurons, towards generating neurons of the glutamatergic lineage. This indicates that Neurog2 can override region specific fate restrictions of adult NSCs. However, once SEZ-derived cells have differentiated into postmitotic neurons, Neurog2 can no longer induce a switch in transmitter phenotype, as neurons retain their GABAergic neuron identity. Thus, there appears to be a restricted time window during the lineage progression from stem cell to neuron during which Neurog 2 can alter the program determining transmitter identity. Our data supporting a stage- and hence cellular context-dependent potency of Neurog2 are in line with a recent study showing that gain-offunction of Neurog2 can bias the balance between deep-layer and upper-layer neurogenesis towards the former in early cortical progenitors, but has a limited capacity to specify early neuronal features in late cortical progenitors (Dennis et al., 2017). Our data also provide additional evidence for Neurog2's instructive role for glutamatergic neurogenesis (Mattar et al., 2008).

\section{Retroviral Neurog2 expression directs dividing SEZ progenitors towards the glutamatergic neuron lineage}

Retroviruses predominantly transduce fast-dividing progenitors both in vitro and in vivo. Thus, by employing a retroviral vector for delivery of Neurog2, expression of the proneural gene should be largely confined to activated stem cells and transit-amplifying progenitors as well as dividing neuroblasts. We found that retrovirus-mediated Neurog2 expression resulted in the acquisition of a glutamatergic neuron identity in vitro, as evidenced by expression of the glutamatergic lineage transcription factors Tbr2 and Tbr1 (Hevner et al., 2006), expression of vesicular glutamate transporter and the development of glutamatergic synapses. In accordance with a fate switch in vivo, Neurog2-expressing cells no longer migrated to the OB via the RMS, but instead differentiated within the SEZ or the adjacent striatum. Of note, a recent study showed SVZ cells can be redirected from their normal migration route and directed towards other brain regions upon co-transduction with retroviruses encoding Neurog2 and Isl1 (Rogelius et al., 2008). Thus, part of the fate switch induced by Neurog2 is an alteration of the migratory program normally under the influence of $D l \times 2$ (Brill et al., 2008). Besides this, differentiated neurons derived from Neurog2-expressing progenitors developed morphological features reminiscent of pyramidal-like neurons. Most importantly a substantial portion of the Neurog2- 
expressing cells co-expressed Tbr2 and Tbr1, demonstrating entry into the glutamatergic neuron lineage (Hevner et al., 2006).

\section{Neurog2 fails to reprogram postmitotic neurons derived from SEZ progenitors towards the} glutamatergic neuron lineage

In sharp contrast to the conspicuous effect of retrovirus-mediated Neurog2 expression, targeting Neurog2 to postmitotic neurons by employing a lentivirus driving transgene expression from the human synapsin promoter (Gascon et al., 2008) failed to alter the already ongoing program of OB interneuron genesis. Lentivirally Neurog2-transduced cells continued to migrate along the RMS to the OB where they took position within the granule cell layer for which most of the adult-generated neurons in the SEZ are destined (Merkle et al., 2014; Merkle et al., 2007). Also, in contrast to Neurog2 expression in progenitors, postmitotic expression did not cause overt changes in morphology and GFP-expressing cells exhibited morphologies highly reminiscent of OB granule neurons. Moreover, primary cultures of neurons derived from SEZ progenitors were GABA immunoreactive when Neurog2 was expressed postmitotically. Finally, both in vitro and in vivo, Neurog2 failed to induce the expression of glutamatergic lineage transcription factors such as Tbr2. In the absence of any evidence for a fate change, it thus appears that Neurog2expressing SEZ-derived neurons maintain the interneuron identity originally acquired during lineage progression from stem cell to neuron. This data argues for the establishment of powerful epigenetics barriers that impede a Neurog2-instructed switch from a GABA-to-glutamatergic transmitter identity.

\section{Specific windows of opportunity for Neurog2-induced programming and reprogramming}

This data suggest that the potency of Neurog2 sharply declines when neurons become postmitotic and is likely linked to changes in the neuronal epigenome during this transition as strongly indicated by the failure to upregulate Tbr2 which is known to be a direct target of Neurog2 (Kovach et al., 2013; Ochiai et al., 2009). Alternatively, or in addition to epigenetic barriers, the effectiveness of Neurog2 action may be curtailed by signaling mechanisms regulating the phosphorylation state (Quan et al., 2016) or the formation of homo- versus heterodimers (Li et al., 2012).

The failure of reprogramming at later stages of the stem cell to neuron lineage progression is even more conspicuous given the capacity of Neurog2 to sequentially induce Tbr2 and Tbr1 expression in astrocytes derived from the early postnatal cerebral cortex and reprogram these into fully functional glutamatergic neurons (Berninger et al., 2007a; Heinrich et al., 2010). Yet, 
also this reprogramming activity of proneural genes such as Neurog2 or Ascll appears to become more restricted with glial maturation (Masserdotti et al., 2015; Ueki et al., 2015) and glia-toneuron conversion by Neurog2 or Ascl1 alone is very limited in the adult central nervous system in vivo (Grande et al., 2013). Remarkably, some of these restrictions can be overcome by allowing for enhanced epigenetic remodeling as recently demonstrated for Ascll-induced reprogramming of Müller glia into bipolar neurons in the lesioned adult mouse retina (Jorstad et al., 2017). Moreover, a recent study identified a critical metabolic checkpoint for Neurog2- and Ascll-induced glia-to-neuron reprogramming which could be negotiated by co-expression of Bcl2 (Gascon et al., 2016). While this check point negotiation appears to work predominantly via interference with reactive oxygen species-induced ferroptosis, it is conceivable that enhanced reprogramming following $B c l 2$ co-expression might also be related to the need of producing larger quantities of particular mitochondrial metabolites required for epigenetic remodeling such as shown in other systems (Wong et al., 2017).

The developmental window-specific actions of Neurog2 on adult SEZ stem and progenitor cells exhibit remarkable parallelism and differences to that of the transcription factor Fezf2. Fezf2 was originally described as a transcription factor to specify the fate of cortical progenitors towards a corticofugal identity during development (Molyneaux et al., 2005). Subsequent work demonstrated that it can reprogram striatal progenitors towards a corticofugal identity in vivo (Rouaux and Arlotta, 2010) thus causing not only a similar transmitter identity switch (from GABAergic to glutamatergic neuron) as described here for Neurog2, but also eliciting a specific neuronal subtype conversion (from medium spiny neuron to corticofugal pyramidal neuron). Of note, Fezf2 reprogramming activity extends into early postmitotic life of a neuron but markedly declines in the course of few days (Rouaux and Arlotta, 2013) arguing for the presence of a critical window of nuclear plasticity that closes with epigenetic changes that occur during neuronal maturation (Amamoto and Arlotta, 2014). More recently, Fezf2 was found to program NSCs in the postnatal and adult SEZ towards a glutamatergic neuron identity in vitro and in vivo (Zuccotti et al., 2014). In conspicuous difference to the findings reported here for forced Neurog2 expression in fast-dividing cells, the Fezf2- induced fate switch was restricted specifically to the stem cell stage and failed to convert both transit-amplifying progenitors or dividing neuroblasts. Moreover, in contrast to the redirection of Neurog2-expressing neurons, progeny of Fezf2-expressing NSCs still migrated to the OB. Thus, Fezf2 and Neurog2 appear to possess distinct temporal windows of opportunity.

Taken together, our data provide evidence for remarkable plasticity within the lineage of adult NSCs. It will be interesting to learn whether this plasticity can be harnessed towards translational 
317 approaches to recruit adult NSC progeny into diseased brain tissue for repair (Benraiss et al., 318 2013; Brill et al., 2009; Gage and Temple, 2013; Saghatelyan et al., 2004). To fully exploit this, 319 it will be of crucial importance to identify the mechanisms underlying the closure of windows of 320 opportunity and thereby terminally sealing neuron fate. 


\section{MATERIAL AND METHODS}

\section{Ethical approval}

All animal procedures were performed in accordance to the Policies on the Use of Animals and Humans in Neuroscience Research, revised and approved by the Society of Neuroscience and the state of Bavaria under license number 55.2-1-54-2531-144/07.

\section{Plasmids and DNA constructs}

Retroviral and lentiviral transduction of SEZ primary cultures was performed 2 hours after plating the cells on glass coverslips, using VSV-G (vesicular stomatitis virus glycoprotein) pseudotyped viruses. For the transduction with retrovirus we used the retroviral vectors RVpCAG-Neurog2-IRES-DsRed and RV-pCAG-IRES-DsRed as described previously (Heinrich et al., 2011). To obtain the lentiviral vector Syn-DsRed-Syn-egfp we re-cloned a fragment containing the cDNA of Neurog2 and the internal ribosomal entry site from the RV-pCAGNeurog2-IRES-DsRed into the lentiviral vector hSyn-DsRed-Syn-egfp (Gascon et al., 2008). In this case, control experiments were performed with the lentiviral vector hSyn-egfp (Gascon et al., 2008).

\section{SEZ primary culture}

Following a previously established protocol by (Costa et al., 2011; Ortega et al., 2011), SEZ cultures were prepared from the lateral wall of the lateral ventricle of young adult ( 8 - 12 weeks) C57/B16 mice (Mus musculus) (Costa et al., 2011; Ortega et al., 2011). Briefly, tissue was dissociated in $0.7 \mathrm{mg} / \mathrm{ml}$ hyaluronic acid (Sigma-Aldrich) and $1.33 \mathrm{mg} / \mathrm{ml}$ trypsin (SigmaAldrich) in Hanks' Balanced Salt Solution (HBSS; Invitrogen) with $2 \mathrm{mM}$ glucose (SigmaAldrich) at $37^{\circ} \mathrm{C}$ for 30 minutes. After this enzymatic treatment, an equal volume of an ice-cold medium consisting of 4\% bovine serum albumin (BSA; Sigma-Aldrich) in Earle's Balanced Salt Solution (EBSS; Invitrogen) buffered with $20 \mathrm{mM}$ HEPES (Invitrogen) was added in order to stop dissociation. Cells were then centrifuged at $200 \mathrm{~g}$ for 5 minutes, re-suspended in ice-cold medium consisting of $0.9 \mathrm{M}$ sucrose (Sigma-Aldrich) in $0.5 \times \mathrm{HBSS}$, and centrifuged for 10 minutes at $750 \mathrm{~g}$. The cell pellet was re-suspended in $2 \mathrm{ml}$ ice-cold medium consisting of $4 \%$ BSA in EBSS buffered with $2 \mathrm{mM}$ HEPES, and the cell suspension was placed on top of $12 \mathrm{ml}$ of the same medium and centrifuged for 7 minutes at $200 \mathrm{~g}$. The resulting cell pellet was resuspended in DMEM/F12 Glutamax (Invitrogen) supplemented with B27 (Invitrogen), 2 mM glutamine (Sigma), 100 units $/ \mathrm{ml}$ penicillin (Invitrogen), $100 \mu \mathrm{g} / \mathrm{ml}$ streptomycin (Invitrogen), buffered with $8 \mathrm{mM}$ HEPES. Finally, cells were plated on poly-d-lysine (Sigma) coated 
coverslips at a density of 200-300 cells per $\mathrm{mm}^{2}$ and, after 2 hours to allow for settlement of the cells, cultures were treated with retroviral or lentiviral vectors for transduction.

\section{Viral vector injections}

Stereotactic injections of retrovirus and lentivirus were performed in 2 - 3 months old C57BL/6 male mice (Mus musculus). Prior to stereotactic injections, mice were anesthetized using Ketamine (100 mg/kg; CP-Pharma) and Xylazine (5 mg/kg; Rompun; Bayer) and placed into a stereotaxic frame. Approximately, $0.5 \mu 1$ viral suspension was injected using a pulled-glass capillary at the following coordinates relative to Bregma: 0.7 (antero-posterior), 1.2 (mediolateral) and 2.1-1.7 (dorso-ventral).

\section{Immunohistochemistry and immunocytochemistry}

Mice were deeply anesthetized using 5\% chloralhydrate (wt/vol) diluted in phosphate buffered saline and then perfused transcardially with saline $(0.9 \%)$, followed by $4 \%$ paraformaldehyde (PFA) (wt/vol) for 30 minutes. After this initial fixation, brains were dissected and post-fixed for at least 2 hours in 4\% PFA. Sagittal brain sections were prepared at a thickness of $50 \mu \mathrm{m}$ using a Thermo Scientific vibrating blade microtome.

For immunohistochemistry, sections were blocked for 90 minutes in TBS containing $0.3 \%$ Triton and 5\% donkey serum. Primary antibodies were diluted in blocking solution and incubated over night at $4{ }^{\circ} \mathrm{C}$ on the sections. In this study, we used antibodies to Tbr1 (Rabbit, 1:100 Abcam, ab31940), Tbr2 (Rabbit, 1:500 Abcam, ab23345), RFP (Rabbit, 1:500 Rockland, 600401379S), Doublecortin (DCX, Goat, 1:300 Santa Cruz Biotechnology, sc-8066) and GFP (Chicken, 1:1000, Aves labs, GFP-1020) The next day samples were washed with TBS and subsequently incubated at room temperature for 1 hour with species-corresponding secondary antibodies made in donkey and conjugated to Cy3 (1:500, Dianova, 711-165-152, 705-165-147), Alexa Fluor 488 (1:200, Invitrogen, A21206, A11055; Jackson Immunoresearch, 703-545-155) and Alexa Fluor 647 (1:500, Invitrogen, A31573, A21447). Sections were washed again in TBS, counterstained with DAPI and mounted with an acqueous mounting medium.

Cultures were fixed in 4\% PFA in PBS for 15 minutes at room temperature and processed for antibody staining as described previously (Ortega et al., 2011).

\section{Electrophysiology}

Perforated patch-clamp recordings were performed as previously described (Heinrich et al., 2011). 


\section{Quantitative and statistical analysis}

391

392

393

394

395

396

397

398

399

400

401

402

403

404

405

406

407

408

409

410

411

412

413

414

415

416

417

418

419

420

421

Images stacks were acquired using a confocal microscope (Olympus FV1000 equipped with x20/0.8 N.A., x40/1.35 and x60/1.42 N.A. oil-immersion objectives). Quantifications of markerpositive cells were performed on single optical sections of an image stack. The percentage of DCX-positive or Tbr2-positive cells was calculated among RFP-positive cells in the SEZ region. For distribution analysis of the cells, RFP-positive/DCX-positive cells were quantified in the SEZ, RMS and OB, and the percentage of cells in each region among total number of RFPpositive/DCX-positive cells was calculated. The number of animals (n) used and the total number of cells is indicated in the text. Data are represented as mean \pm s.d. Statistical analysis was performed using SPSS Statistics 22 software. The statistical tests used and p values are indicated in the figure legends.

\section{Acknowledgements}

We are grateful to Dr. Magdalena Götz for support throughout the project.

\section{Competing interests}

The authors declare no competing financial interests.

\section{Author contributions}

S.P., L.M.M and M.S.B designed and performed experiments, interpreted and analyzed results, and wrote the manuscript. F.O. and M.K. designed and performed experiments. S.G. conceptualized, designed and performed experiments, interpreted and analyzed results, and wrote the manuscript. B.B. conceptualized the study and experiments, interpreted results, and wrote the manuscript. All authors discussed the manuscript.

\section{Funding}

This work was supported by grants from the Deutsche Forschungsgemeinschaft (CRC1080, INST 247/695-1), Belgian Science Policy Organization (Interuniversity attraction pole P7/20 "Wibrain") and ERA-NET NEURON (01EW1604) to B.B., and the Bavarian State Ministry of Sciences, Research and the Arts consortium "ForIPS" to M.K. and B.B.

\section{References}


Amamoto, R. and Arlotta, P. (2014). Development-inspired reprogramming of the mammalian central nervous system. Science 343, 1239882.

Arlotta, P. and Berninger, B. (2014). Brains in metamorphosis: reprogramming cell identity within the central nervous system. Curr Opin Neurobiol 27, 208-214.

Azim, K., Hurtado-Chong, A., Fischer, B., Kumar, N., Zweifel, S., Taylor, V. and Raineteau, O. (2015). Transcriptional Hallmarks of Heterogeneous Neural Stem Cell Niches of the Subventricular Zone. Stem Cells 33, 2232-2242.

Benraiss, A., Toner, M. J., Xu, Q., Bruel-Jungerman, E., Rogers, E. H., Wang, F., Economides, A. N., Davidson, B. L., Kageyama, R., Nedergaard, M., et al. (2013). Sustained mobilization of endogenous neural progenitors delays disease progression in a transgenic model of Huntington's disease. Cell Stem Cell 12, 787-799.

Berninger, B., Costa, M. R., Koch, U., Schroeder, T., Sutor, B., Grothe, B. and Gotz, M. (2007a). Functional properties of neurons derived from in vitro reprogrammed postnatal astroglia. $J$ Neurosci 27, 8654-8664.

Berninger, B., Guillemot, F. and Gotz, M. (2007b). Directing neurotransmitter identity of neurones derived from expanded adult neural stem cells. Eur J Neurosci 25, 2581-2590.

Brill, M. S., Ninkovic, J., Winpenny, E., Hodge, R. D., Ozen, I., Yang, R., Lepier, A., Gascon, S., Erdelyi, F., Szabo, G., et al. (2009). Adult generation of glutamatergic olfactory bulb interneurons. Nat Neurosci 12, 1524-1533.

Brill, M. S., Snapyan, M., Wohlfrom, H., Ninkovic, J., Jawerka, M., Mastick, G. S., Ashery-Padan, R., Saghatelyan, A., Berninger, B. and Gotz, M. (2008). A dlx2- and pax6-dependent transcriptional code for periglomerular neuron specification in the adult olfactory bulb. $J$ Neurosci 28, 6439-6452.

Chen, X., Lepier, A., Berninger, B., Tolkovsky, A. M. and Herbert, J. (2012). Cultured subventricular zone progenitor cells transduced with neurogenin-2 become mature glutamatergic neurons and integrate into the dentate gyrus. PloS one 7, e31547.

Costa, M. R., Ortega, F., Brill, M. S., Beckervordersandforth, R., Petrone, C., Schroeder, T., Gotz, M. and Berninger, B. (2011). Continuous live imaging of adult neural stem cell division and lineage progression in vitro. Development 138, 1057-1068.

Dennis, D. J., Wilkinson, G., Li, S., Dixit, R., Adnani, L., Balakrishnan, A., Han, S., Kovach, C., Gruenig, N., Kurrasch, D. M., et al. (2017). Neurog2 and Ascl1 together regulate a postmitotic derepression circuit to govern laminar fate specification in the murine neocortex. Proc Natl Acad Sci U S A 114, E4934-E4943.

Doetsch, F., Petreanu, L., Caille, I., Garcia-Verdugo, J. M. and Alvarez-Buylla, A. (2002). EGF converts transit-amplifying neurogenic precursors in the adult brain into multipotent stem cells. Neuron 36, 1021-1034.

Gabay, L., Lowell, S., Rubin, L. L. and Anderson, D. J. (2003). Deregulation of dorsoventral patterning by FGF confers trilineage differentiation capacity on CNS stem cells in vitro. Neuron $\mathbf{4 0 , 4 8 5 -}$ 499.

Gage, F. H. and Temple, S. (2013). Neural stem cells: generating and regenerating the brain. Neuron 80, 588-601.

Gascon, S., Murenu, E., Masserdotti, G., Ortega, F., Russo, G. L., Petrik, D., Deshpande, A., Heinrich, C., Karow, M., Robertson, S. P., et al. (2016). Identification and Successful Negotiation of a Metabolic Checkpoint in Direct Neuronal Reprogramming. Cell Stem Cell 18, 396-409.

Gascon, S., Paez-Gomez, J. A., Diaz-Guerra, M., Scheiffele, P. and Scholl, F. G. (2008). Dual-promoter lentiviral vectors for constitutive and regulated gene expression in neurons. I Neurosci Methods 168, 104-112.

Grande, A., Sumiyoshi, K., Lopez-Juarez, A., Howard, J., Sakthivel, B., Aronow, B., Campbell, K. and Nakafuku, M. (2013). Environmental impact on direct neuronal reprogramming in vivo in the adult brain. Nat Commun 4, 2373. 
Hack, M. A., Saghatelyan, A., de Chevigny, A., Pfeifer, A., Ashery-Padan, R., Lledo, P. M. and Gotz, M. (2005). Neuronal fate determinants of adult olfactory bulb neurogenesis. Nat Neurosci 8, 865-872.

Hack, M. A., Sugimori, M., Lundberg, C., Nakafuku, M. and Gotz, M. (2004). Regionalization and fate specification in neurospheres: the role of Olig2 and Pax6. Mol Cell Neurosci 25, 664-678.

Heinrich, C., Blum, R., Gascon, S., Masserdotti, G., Tripathi, P., Sanchez, R., Tiedt, S., Schroeder, T., Gotz, M. and Berninger, B. (2010). Directing astroglia from the cerebral cortex into subtype specific functional neurons. PLoS Biol 8, e1000373.

Heinrich, C., Gascon, S., Masserdotti, G., Lepier, A., Sanchez, R., Simon-Ebert, T., Schroeder, T., Gotz, M. and Berninger, B. (2011). Generation of subtype-specific neurons from postnatal astroglia of the mouse cerebral cortex. Nat Protoc 6, 214-228.

Hevner, R. F., Hodge, R. D., Daza, R. A. and Englund, C. (2006). Transcription factors in glutamatergic neurogenesis: conserved programs in neocortex, cerebellum, and adult hippocampus. Neurosci Res 55, 223-233.

Jorstad, N. L., Wilken, M. S., Grimes, W. N., Wohl, S. G., VandenBosch, L. S., Yoshimatsu, T., Wong, R. O., Rieke, F. and Reh, T. A. (2017). Stimulation of functional neuronal regeneration from Muller glia in adult mice. Nature.

Kovach, C., Dixit, R., Li, S., Mattar, P., Wilkinson, G., Elsen, G. E., Kurrasch, D. M., Hevner, R. F. and Schuurmans, C. (2013). Neurog2 simultaneously activates and represses alternative gene expression programs in the developing neocortex. Cereb Cortex 23, 1884-1900.

Li, S., Mattar, P., Zinyk, D., Singh, K., Chaturvedi, C. P., Kovach, C., Dixit, R., Kurrasch, D. M., Ma, Y. C., Chan, J. A., et al. (2012). GSK3 temporally regulates neurogenin 2 proneural activity in the neocortex. J Neurosci 32, 7791-7805.

Lim, D. A. and Alvarez-Buylla, A. (2014). Adult neural stem cells stake their ground. Trends Neurosci 37, 563-571.

Lois, C., Garcia-Verdugo, J. M. and Alvarez-Buylla, A. (1996). Chain migration of neuronal precursors. Science 271, 978-981.

Masserdotti, G., Gillotin, S., Sutor, B., Drechsel, D., Irmler, M., Jorgensen, H. F., Sass, S., Theis, F. J., Beckers, J., Berninger, B., et al. (2015). Transcriptional Mechanisms of Proneural Factors and REST in Regulating Neuronal Reprogramming of Astrocytes. Cell Stem Cell 17, 74-88.

Mattar, P., Langevin, L. M., Markham, K., Klenin, N., Shivji, S., Zinyk, D. and Schuurmans, C. (2008). Basic helix-loop-helix transcription factors cooperate to specify a cortical projection neuron identity. Mol Cell Biol 28, 1456-1469.

Merkle, F. T., Fuentealba, L. C., Sanders, T. A., Magno, L., Kessaris, N. and Alvarez-Buylla, A. (2014). Adult neural stem cells in distinct microdomains generate previously unknown interneuron types. Nat Neurosci 17, 207-214.

Merkle, F. T., Mirzadeh, Z. and Alvarez-Buylla, A. (2007). Mosaic organization of neural stem cells in the adult brain. Science 317, 381-384.

Molyneaux, B. J., Arlotta, P., Hirata, T., Hibi, M. and Macklis, J. D. (2005). Fezl is required for the birth and specification of corticospinal motor neurons. Neuron 47, 817-831.

Ochiai, W., Nakatani, S., Takahara, T., Kainuma, M., Masaoka, M., Minobe, S., Namihira, M., Nakashima, K., Sakakibara, A., Ogawa, M., et al. (2009). Periventricular notch activation and asymmetric Ngn2 and Tbr2 expression in pair-generated neocortical daughter cells. Mol Cell Neurosci 40, 225-233.

Ortega, F., Costa, M. R., Simon-Ebert, T., Schroeder, T., Gotz, M. and Berninger, B. (2011). Using an adherent cell culture of the mouse subependymal zone to study the behavior of adult neural stem cells on a single-cell level. Nat Protoc 6, 1847-1859.

Ortega, F., Gascon, S., Masserdotti, G., Deshpande, A., Simon, C., Fischer, J., Dimou, L., Chichung Lie, D., Schroeder, T. and Berninger, B. (2013). Oligodendrogliogenic and neurogenic adult subependymal zone neural stem cells constitute distinct lineages and exhibit differential responsiveness to Wnt signalling. Nat Cell Biol 15, 602-613. 
Quan, X. J., Yuan, L., Tiberi, L., Claeys, A., De Geest, N., Yan, J., van der Kant, R., Xie, W. R., Klisch, T. J., Shymkowitz, J., et al. (2016). Post-translational Control of the Temporal Dynamics of Transcription Factor Activity Regulates Neurogenesis. Cell 164, 460-475.

Rogelius, N., Hebsgaard, J. B., Lundberg, C. and Parmar, M. (2008). Reprogramming of neonatal SVZ progenitors by islet-1 and neurogenin-2. Mol Cell Neurosci 38, 453-459.

Rouaux, C. and Arlotta, P. (2010). Fezf2 directs the differentiation of corticofugal neurons from striatal progenitors in vivo. Nat Neurosci 13, 1345-1347.

---- (2013). Direct lineage reprogramming of post-mitotic callosal neurons into corticofugal neurons in vivo. Nat Cell Biol 15, 214-221.

Saghatelyan, A., de Chevigny, A., Schachner, M. and Lledo, P. M. (2004). Tenascin-R mediates activity-dependent recruitment of neuroblasts in the adult mouse forebrain. Nat Neurosci 7 , 347-356.

Sequerra, E. B. (2014). Subventricular zone progenitors in time and space: generating neuronal diversity. Front Cell Neurosci 8, 434.

Ueki, Y., Wilken, M. S., Cox, K. E., Chipman, L., Jorstad, N., Sternhagen, K., Simic, M., Ullom, K., Nakafuku, M. and Reh, T. A. (2015). Transgenic expression of the proneural transcription factor Ascl1 in Muller glia stimulates retinal regeneration in young mice. Proc Natl Acad Sci U S A 112, 13717-13722.

Wong, B. W., Wang, X., Zecchin, A., Thienpont, B., Cornelissen, I., Kalucka, J., Garcia-Caballero, M., Missiaen, R., Huang, H., Bruning, U., et al. (2017). The role of fatty acid beta-oxidation in lymphangiogenesis. Nature 542, 49-54.

Zuccotti, A., Le Magueresse, C., Chen, M., Neitz, A. and Monyer, H. (2014). The transcription factor Fezf2 directs the differentiation of neural stem cells in the subventricular zone toward a cortical phenotype. Proc Natl Acad Sci U S A 111, 10726-10731. 


\section{FIGURE LEGENDS}

Figure 1. Retrovirus-mediated expression of Neurog2 induces a glutamatergic phenotype in adult SEZ progenitors. (A), Scheme of the retroviral vectors (RV) CAG-Neurog2-IRES-DsRed and control CAG-IRES-DsRed used in this study. (B), Immunocytochemistry of SEZ cultures 30 days after transduction with the RV CAG-Neurog2-IRES-DsRed or the corresponding control RV CAG-IRES-DsRed. Note that Neurog2-expressing cells (right panel; red) are immunopositive for vGluT (green) and MAP2 (blue), indicating a neuronal glutamatergic phenotype. In contrast, cells infected with the DsRed-only-encoding vector (left panel; red) remain negative for vGluT. (C), Patch-clamp recording revealing the glutamatergic identity of neurons derived from Neurog2-expressing SEZ progenitors. Micrographs show Neurog2expressing cells as indicated by the DsRed fluorescence (left panel) and the recording configuration (right panel). The cell marked with the black asterisk expresses Neurog2 (DsRedpositive in the left panel), while the cell marked by the white asterisk is untransduced (DsRednegative in the left panel). Following step depolarisation of the untransduced cell a postsynaptic response is elicited in the Neurog2-expressing cell that reversed at a membrane potential more negative than $-40 \mathrm{mV}$ characteristic of GABAergic transmission and consistent with the GABAergic nature of the untransduced neuron (left trace). In contrast, stimulation of the Neurog2-expressing neuron resulted in a postsynaptic response that was abolished by the AMPA/kainate receptor antagonist CNQX, demonstrating the glutamatergic neuron identity of the Neurog2-expressing neuron (right trace). Scale bars: $10 \mu \mathrm{m}(\mathrm{B}), 20 \mu \mathrm{m}(\mathrm{C})$.

Figure 2. Retrovirus-mediated Neurog2 expression in vivo alters the migration behaviour of SEZ progenitors. (A-D), Sagittal view of an adult mouse brain depicting SEZ cells transduced with the control RV CAG-IRES-DsRed (red) (B), migrating through the RMS (C) and reaching the OB (D). (E-H), Micrographs showing cells transduced with the RV CAG-Neurog2-IRESDsRed (red). At 7 DPI, only few transduced neuroblasts partially entered the RMS (G) and failed to reach the core of the $\mathrm{OB}(\mathrm{H})$. They instead remained stationary in the anterior portion of the SEZ (F). (I), Quantification of the number of DCX-positive/DsRed-positive infected cells in the SVZ, RMS and OB at 7 DPI. Error bars indicate mean \pm s.d., $n=3$ /group. ${ }^{*} \mathrm{p}^{<} 0.05,{ }^{*} \mathrm{p}^{<} 0.01$, $* * * p^{<} 0.001$, One-Way ANOVA followed by Tukey's HSD post-hoc test. Scale bars: $1 \mathrm{~mm}$ (A,E), $500 \mu \mathrm{m}(\mathrm{B}-\mathrm{D}$ and $\mathrm{F}-\mathrm{H})$.

Figure 3. Retrovirus-mediated Neurog 2 expression induces a glutamatergic neuron identity in vivo. (A,B), Micrographs depicting transduced SEZ cells with either control RV (red) (A) or RV CAG-Neurog2-IRES-DsRed (red) (B) co-expressing DCX (green) at 7 DPI. Note that some 
Neurog2-transduced neuroblasts invade the adjacent striatum. (C,D) Higher-power images showing a Neurog2-transduced cell with neuronal morphology (C) and spines (D). (E,F), Micrographs depicting transduced cells immune-positive for DCX (green) (G), Quantification of the percentage of DCX-positive cells among infected cells. Error bars indicate mean \pm s.d., n.s, non-significant, t-test. (H-I), Micrographs showing the expression of the transcription factor Tbr2 (green) in SEZ cells forced to express Neurog2 (I), while cells infected with the control retrovirus lack Tbr2 expression $(\mathrm{H})$. (J), Quantification shows that more than one-third of Neurog2-expressing cells up-regulated Tbr2. Error bars indicate mean \pm s.d., n=3/group. ${ }^{* *} \mathrm{p}^{<} 0.01$, t-test. (K), Micrograph depicting Tbr1 expression (green) in cells transduced with the RV CAG-Neurog2-IRES-DsRed (red). Scale bars: $100 \mu \mathrm{m}$ (A,B), $10 \mu \mathrm{m}$ (C), $5 \mu \mathrm{m}$ (D), $25 \mu \mathrm{m}$ (E,F), $20 \mu \mathrm{m}(\mathrm{H}, \mathrm{I}, \mathrm{K})$.

Figure 4. Forced Neurog2 expression fails to induce a glutamatergic phenotype in postmitotic neurons derived from SEZ progenitors. (A), Scheme of lentiviral vectors hSynNeurog2-IRES-egfp and hSyn-egfp used. (B), Micrographs show the expression of Neurog2 protein (red) and GFP (green) in primary cortical cultures from embryonic day E 14, 6 days after transduction with the hSyn-Neurog2-IRES-egfp lentiviral vector (right panel), or the control lentiviral vector encoding only GFP (left panel). Note that the human synapsin (hSyn) promoter drives selective expression of the transgene in Tuj-1 neurons (blue). (C), In adult SEZ primary cultures, most of the neuroblasts transduced with the Syn-Neurog2-IRES- $g f p$ do not differentiate into vGluT-positive (red) glutamatergic neurons at 30 DPI. (D,E) Example micrographs of Neurog2-expressing neurons (hSyn-Neurog2-IRES-egfp) (D) and control neurons (hSyn-egfp) (E) exhibiting vGluT immunoreactivity, indicative of the rare presence of endogenous glutamatergic neurons. Scale bars: $50 \mu \mathrm{m}(\mathrm{B}-\mathrm{E})$.

Figure 5. Neurog2 induces hallmarks of the glutamatergic lineage when expressed in dividing progenitors but not in young postmitotic neurons. (A), Cells from SEZ cultures infected with the RV CAG-Neurog2-IRES-DsRed differentiate into Tuj-1-positive neurons expressing the early glutamatergic transcription factor Tbr2 (right panel, arrows) at 7 DPI, while those cells infected with the lentiviral hSyn-egfp control vector fail to express Tbr2 (left panel). Note the cluster of non-transduced progenitors expressing Tbr2 (arrowheads) occurring at low frequency in SEZ cultures. (B), At later time points (9 DPI), neurons derived from progenitors transduced with RV CAG-Neurog2-IRES-DsRed also express Tbr1 (arrows). (C), Tuj-1-positive SEZ neuroblasts transduced with RV CAG-Neurog2-IRES-DsRed are devoid of GABA immunoreactivity at 7 DPI (right panel, arrowheads) while neuroblasts transduced with the 
614 lentiviral vector Syn-neurog2-IRES-egfp remain immunoreactive for GABA (left panel, arrows).

615 (D), Quantification of the proportions of transduced cells immunoreactive for Tbr2 or GABA at 6167 DPI following retro- or lentiviral-mediated expression of Neurog2. Error bars indicate mean \pm s.d. $* * p^{<} 0.01, * * * p^{<} 0.001, t$ test. Scale bars: $60 \mu \mathrm{m}(\mathrm{A}-\mathrm{C})$.

Figure 6. Expression of Neurog2 fails to induce a glutamatergic phenotype in postmitotic neurons derived from SEZ progenitors in vivo. (A), Quantification of the number of DCXpositive/GFP-positive LV-transduced cells in the SVZ, RMS and OB shows that the migration of SEZ cells to the OB is not affected by post-mitotic induction of Neurog2. Error bars indicate mean \pm s.d., $n=3$ /group, n.s., non-significant, One-Way ANOVA followed by Tukey's HSD post-hoc test. (B), Micrograph showing neuroblasts (DCX, in red) leaving the SEZ and expressing GFP (in green), indicative of lentiviral transduction with Neurog2 and hSyn-promoter activity in these cells. (C,D), Micrographs of the granule layer of the OB showing lentivirally transduced cells (green) migrating radially and integrating as granule and or periglomerular neurons (PGN, insets, DCX red) at 10 DPI. (E-F), SEZ cells transduction with the hSyn-egfp control (green) and the hSyn-Neurog2-IRES-egfp (green) lentiviruses do not result in Tbr2 expression (red) in periglomerular (PGN, insets) or granule neurons. Scale bars: $50 \mu \mathrm{m}$ (B,C,D,E,F), $10 \mu \mathrm{m}$ (insets in C,D,E,F). 
bioRxiv preprint doi: https://doi.org/10.1101/171686; this version posted August 3, 2017. The copyright holder for this preprint (which was not certified by peer review) is the author/funder. All rights reserved. No reuse allowed without permission.

A

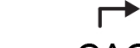

Neurog2 IRES DsRed

CAG

\section{DsRed}

B
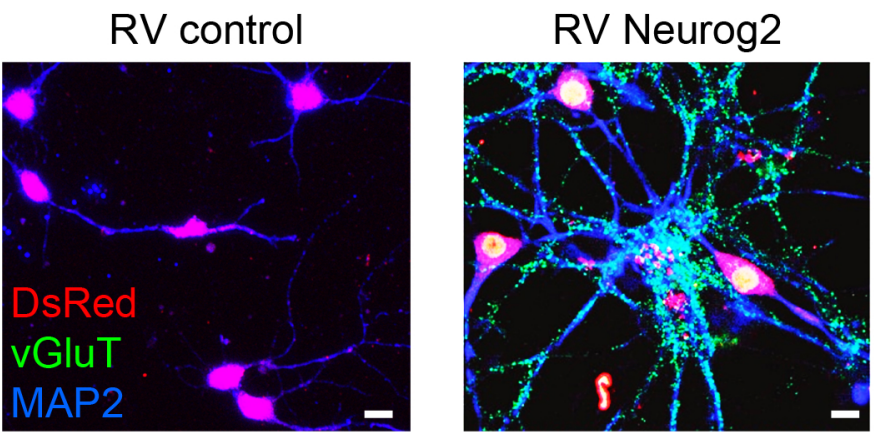

C
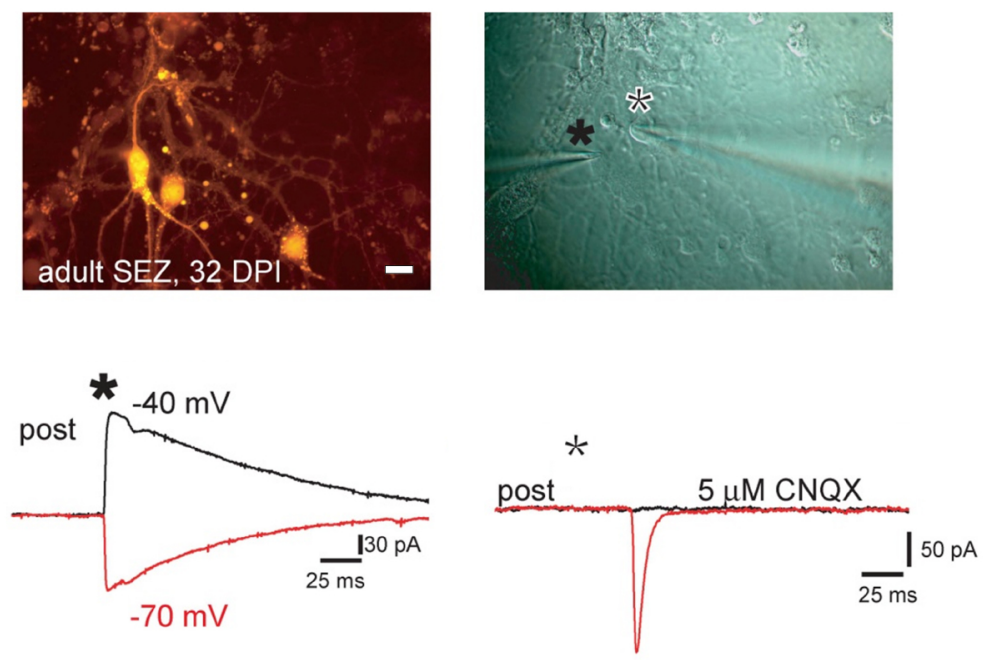
bioRxiv preprint doi: https://doi.org/10.1101/171686; this version posted August 3, 2017. The copyright holder for this preprint (which was not certified by peer review) is the author/funder. All rights reserved. No reuse allowed without permission.

RV control
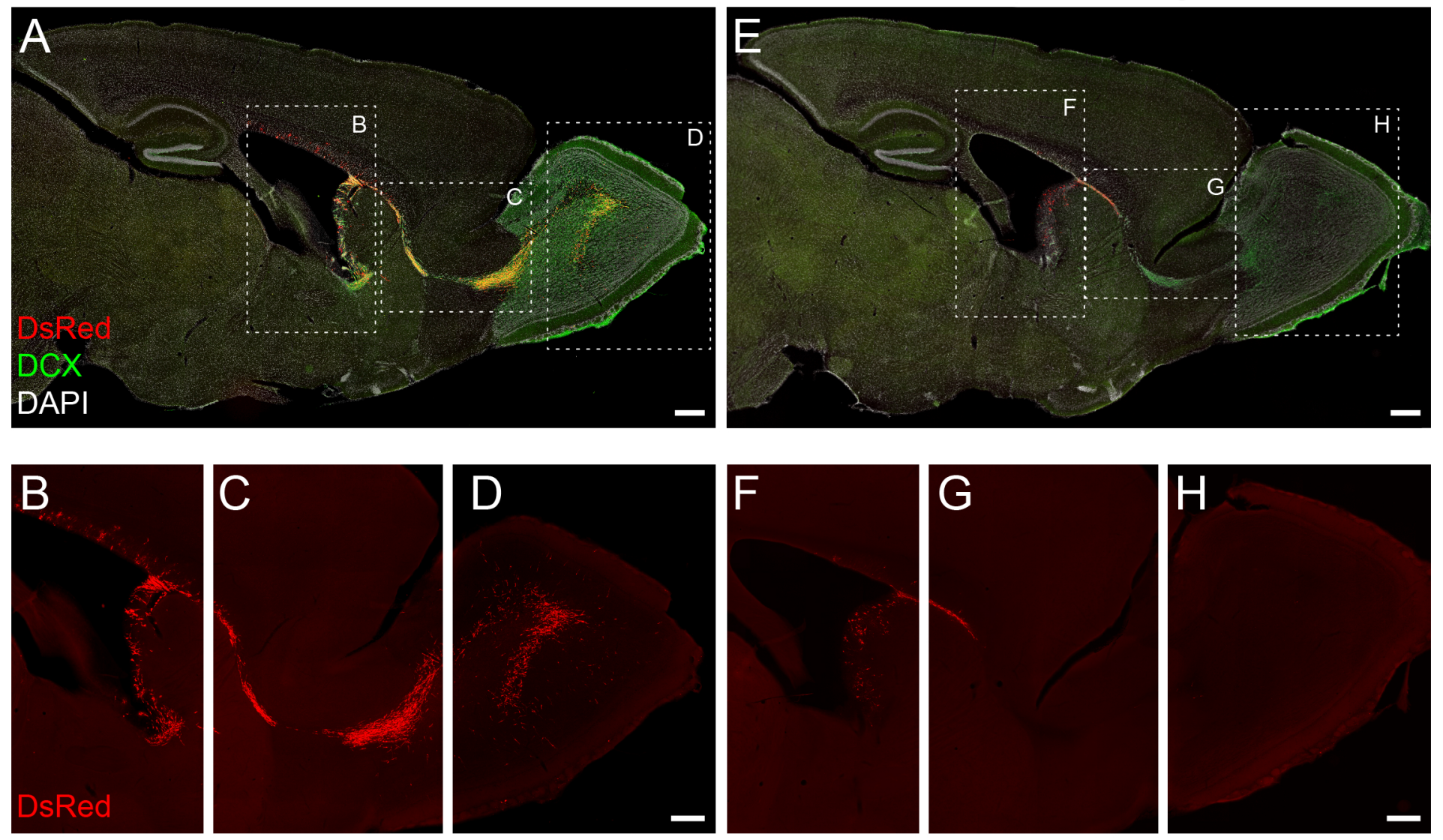

\section{RV Neurog2}

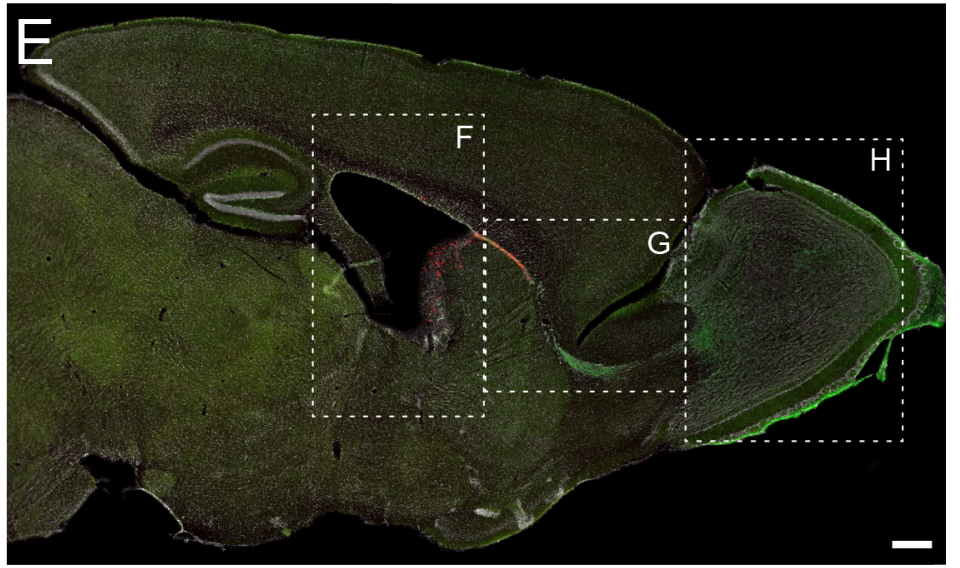

RV control

RV Neurog2

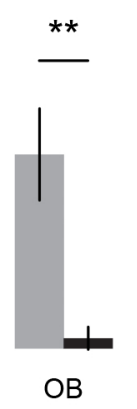


bioRxiv preprint doi: https://doi.org/10.1101/171686; this version posted August 3, 2017. The copyright holder for this preprint (which was

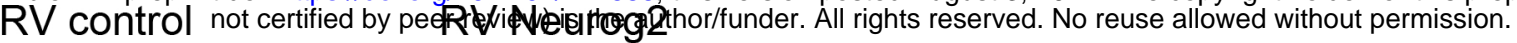
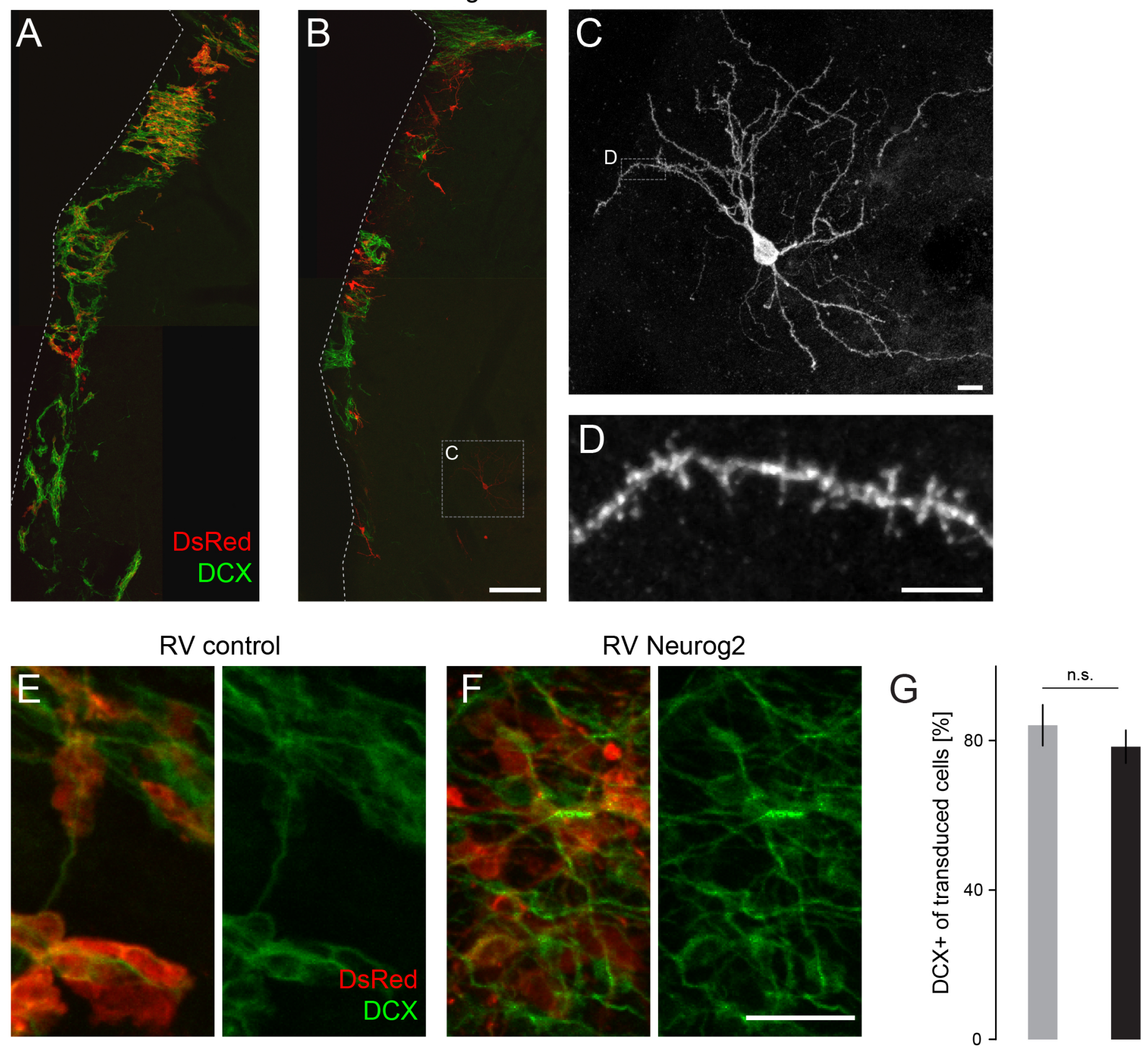

control Neurog2
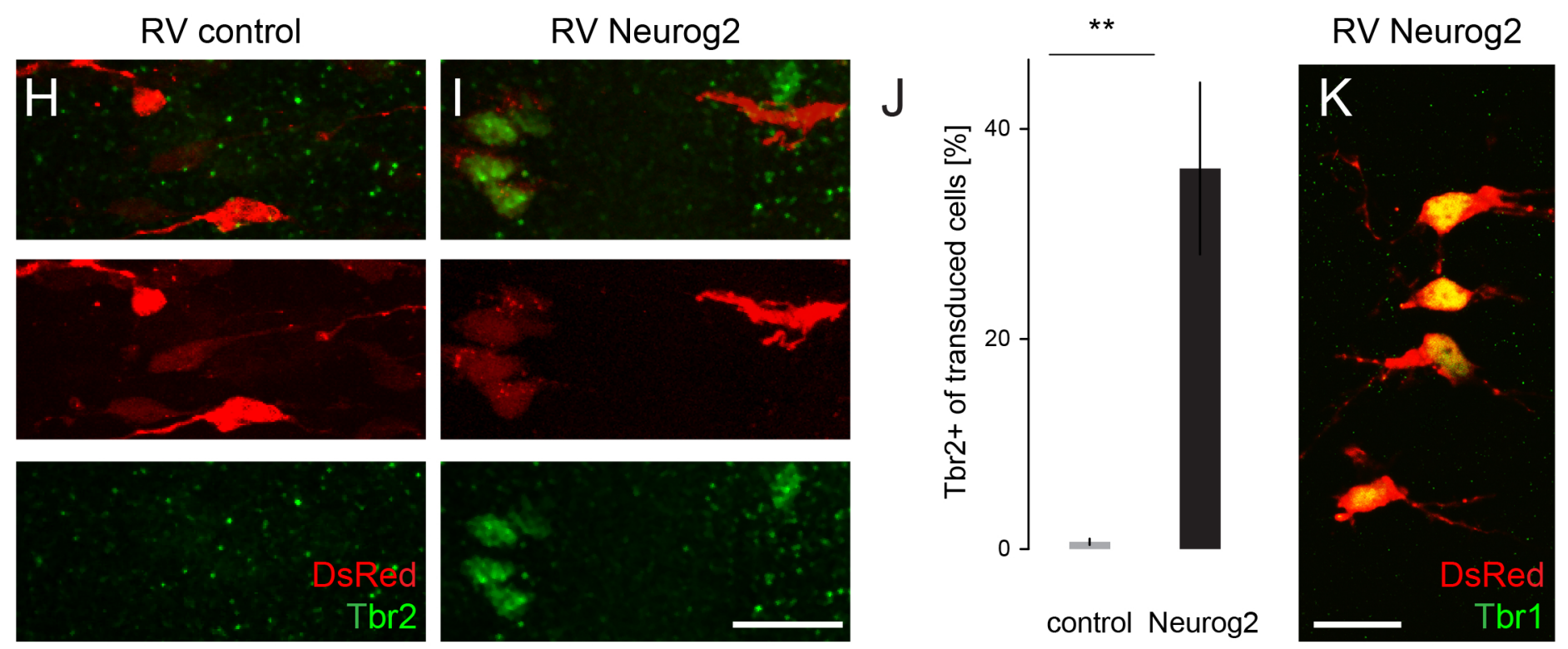

Figure 3 
A

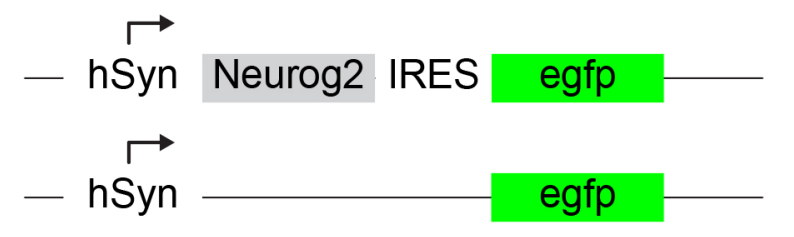

LV control
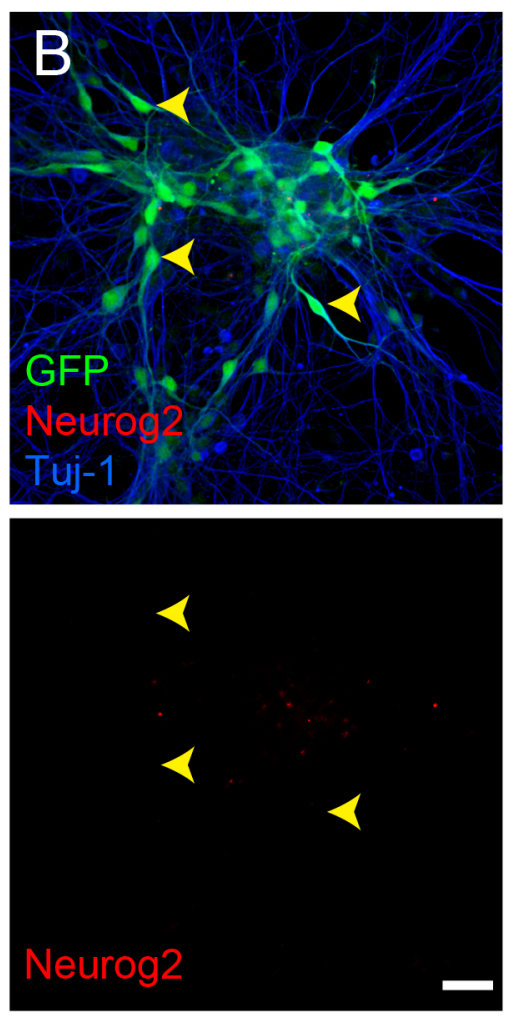

LV Neurog2
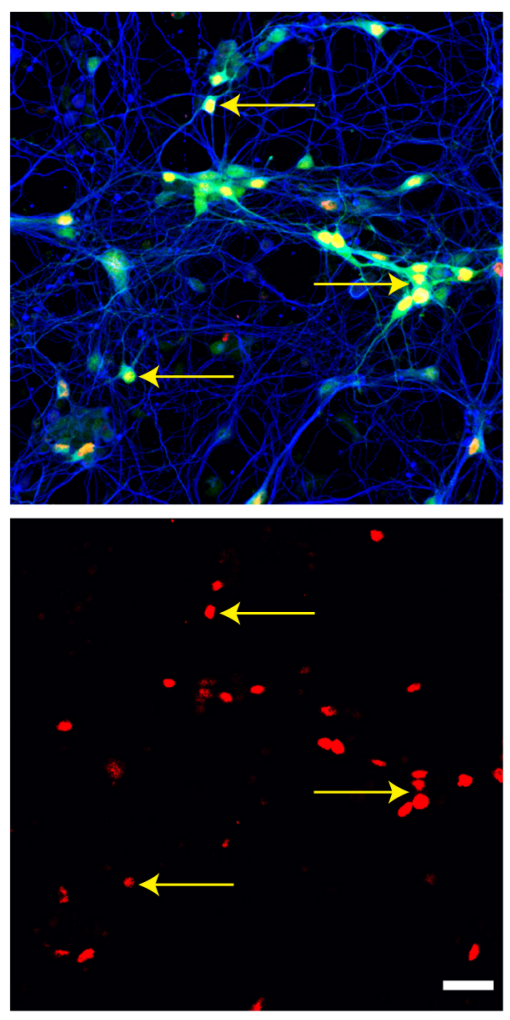

LV Neurog2
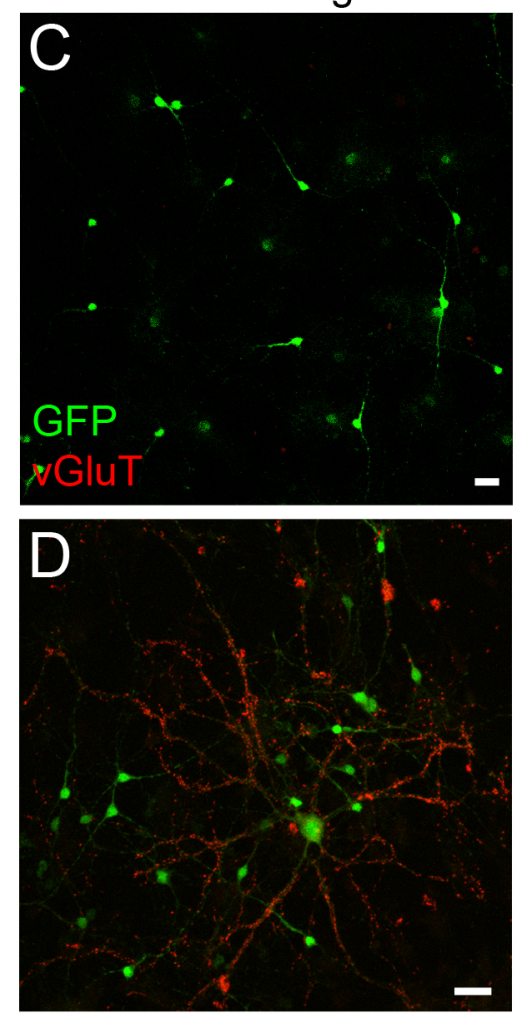

LV control

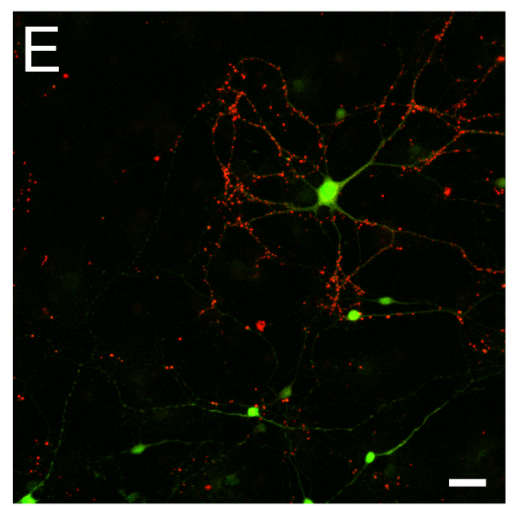


bioRxiv preprint doi: https://doi.org/10.1101/171686; this version posted August 3, 2017. The copyright holder for this preprint (which was not certified by peer review) is the author/funder. All rights reserved. No reuse allowed without permission.

A
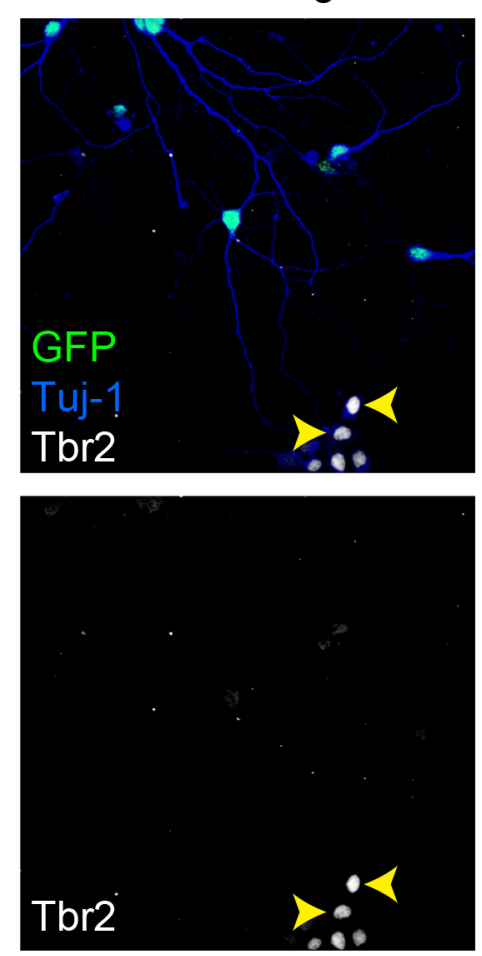

C
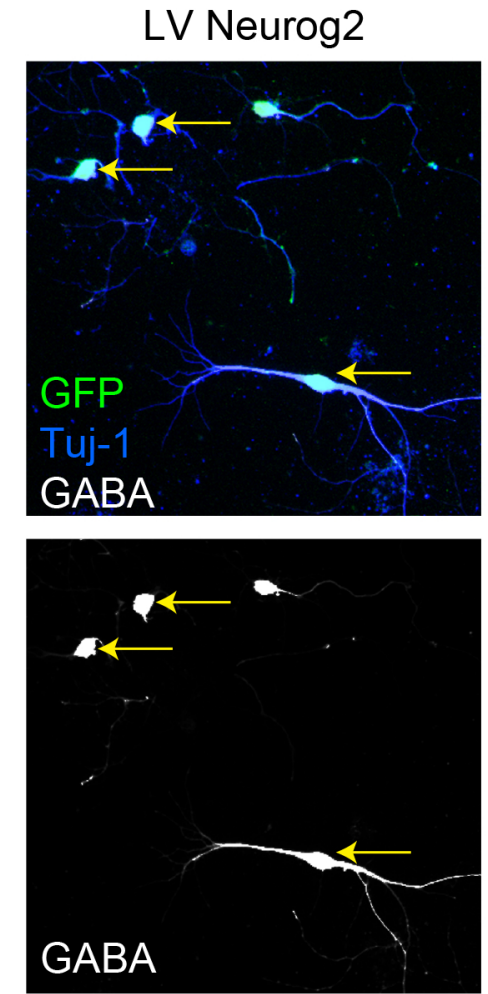
RV Neurog2
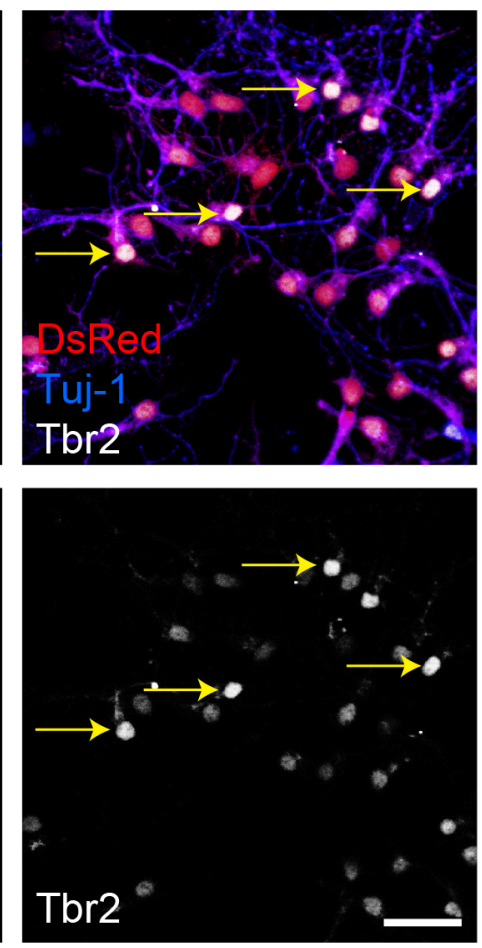

RV Neurog2
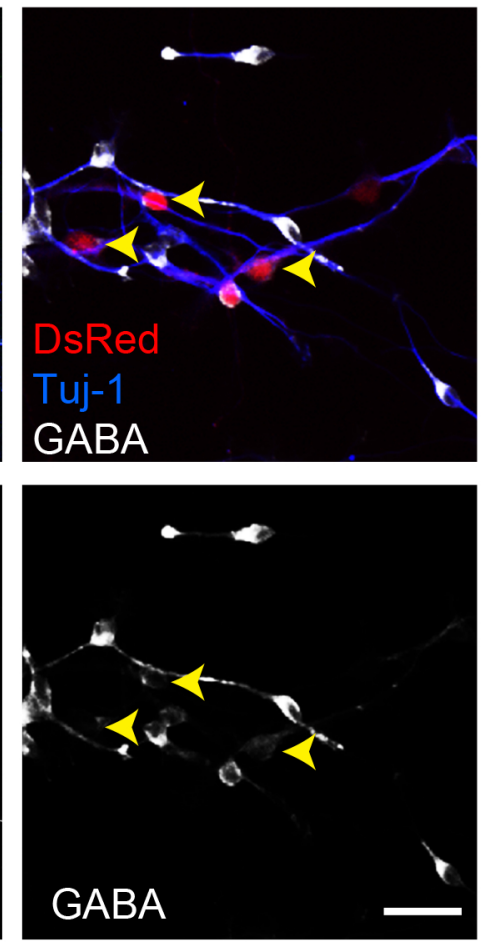

B RV Neurog2
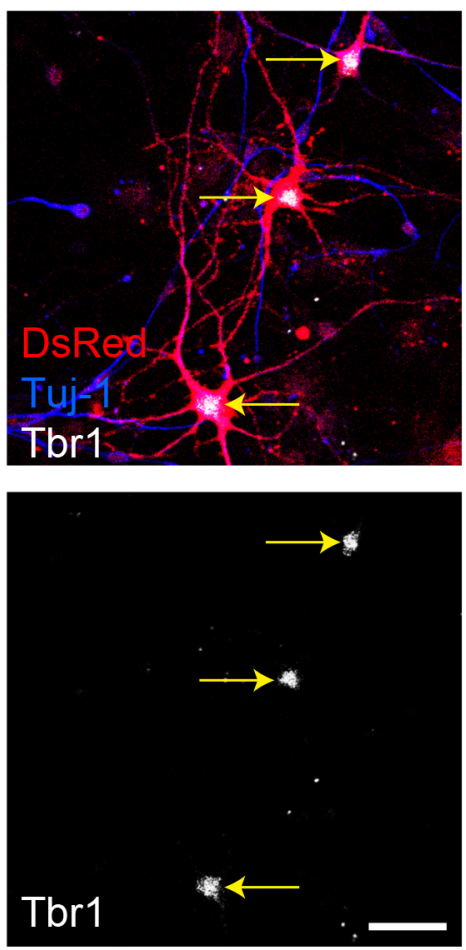

$\mathrm{D}$

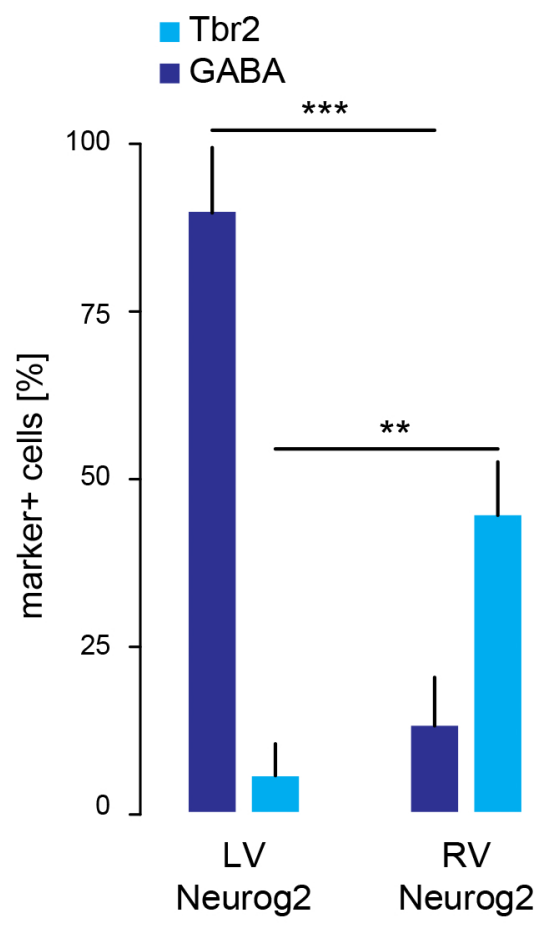


bioRxiv preprint doi: https://doi.org/10.1101/171686; this version posted August 3, 2017. The copyright holder for this preprint (which was not certified by peer review) is the author/funder. All rights reselvbd. Netepogetlowed without permission.
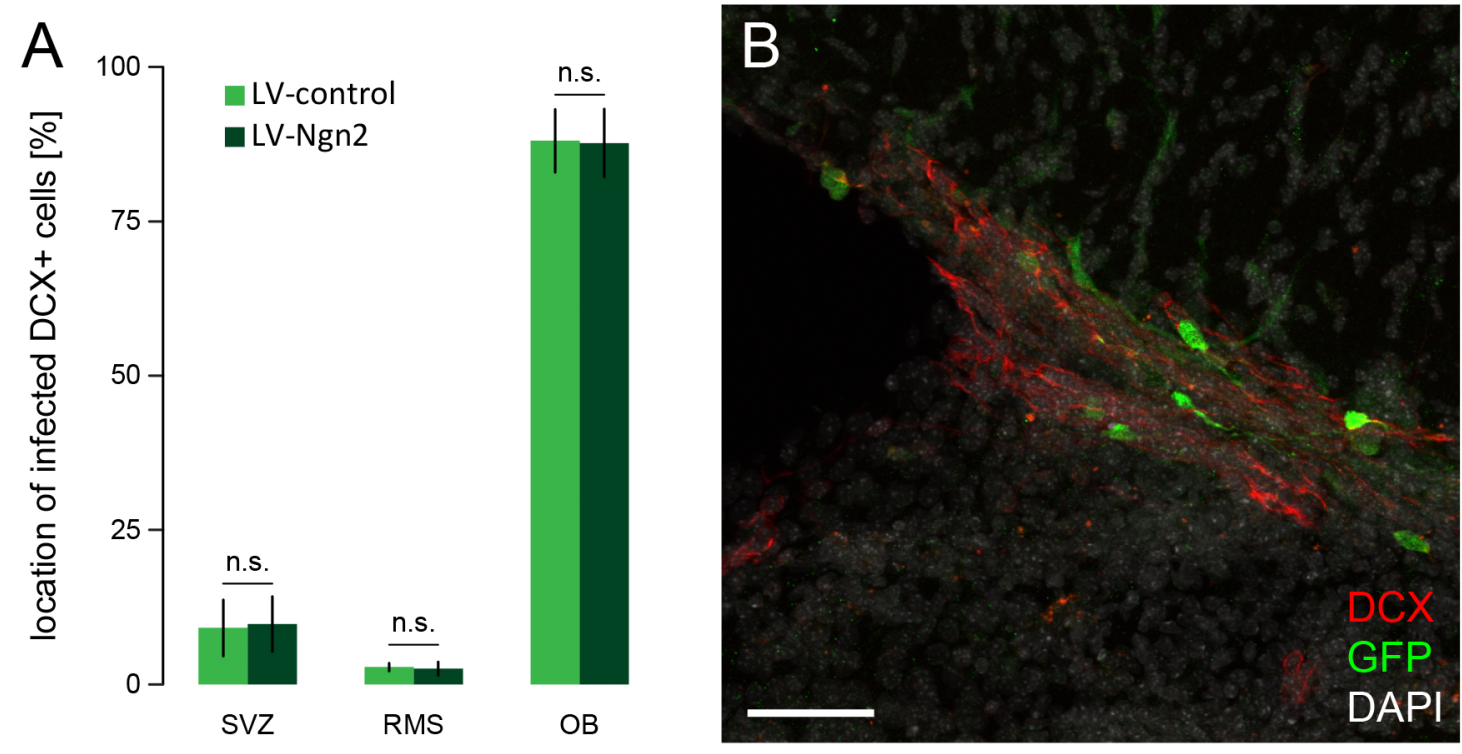

LV control

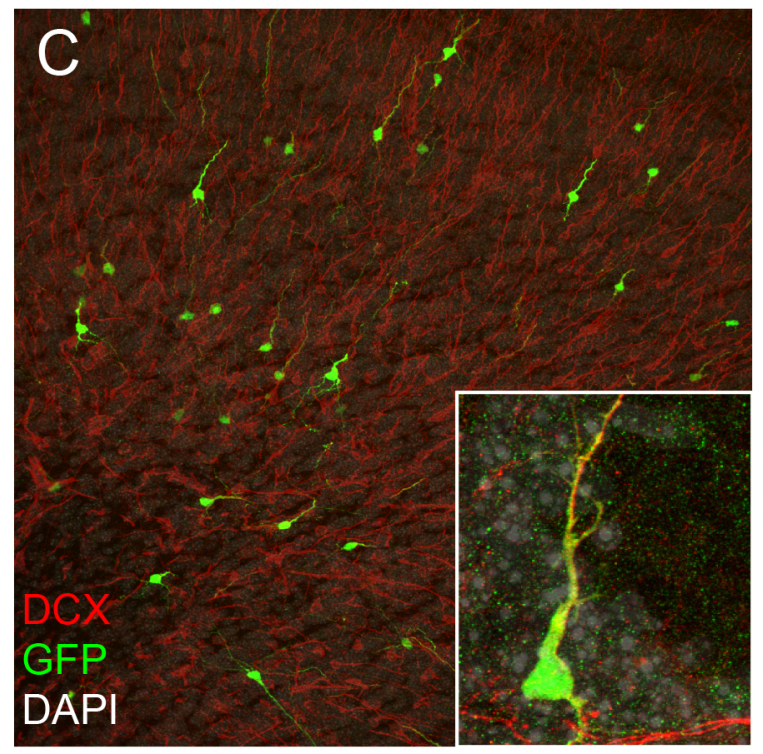

\section{Neurog2}

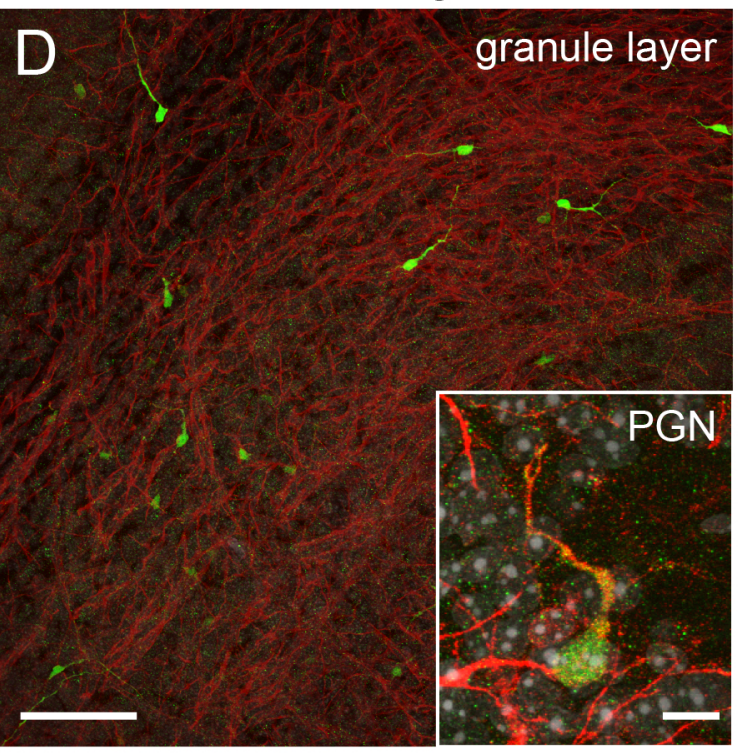

LV control

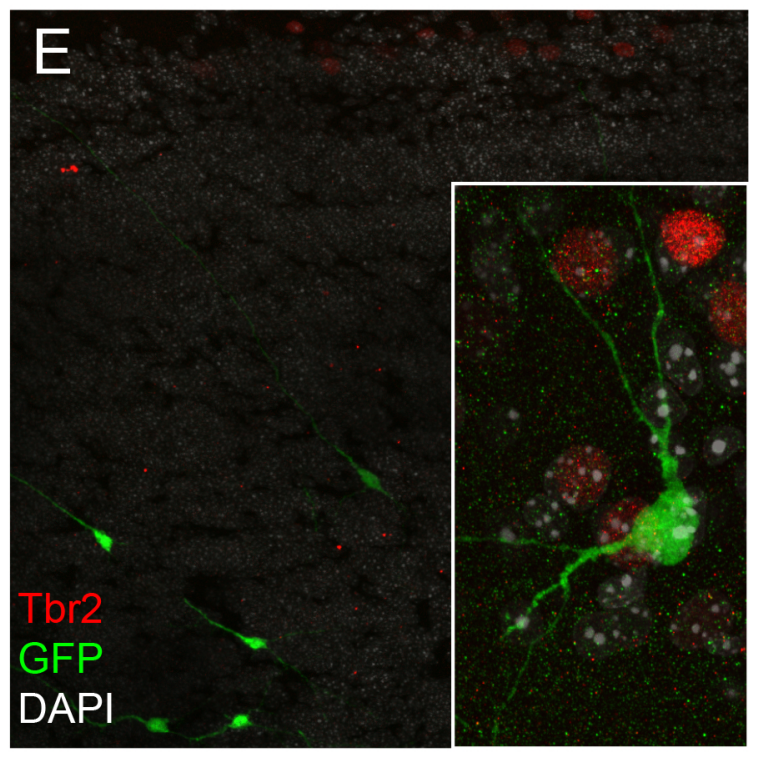

LV Neurog2

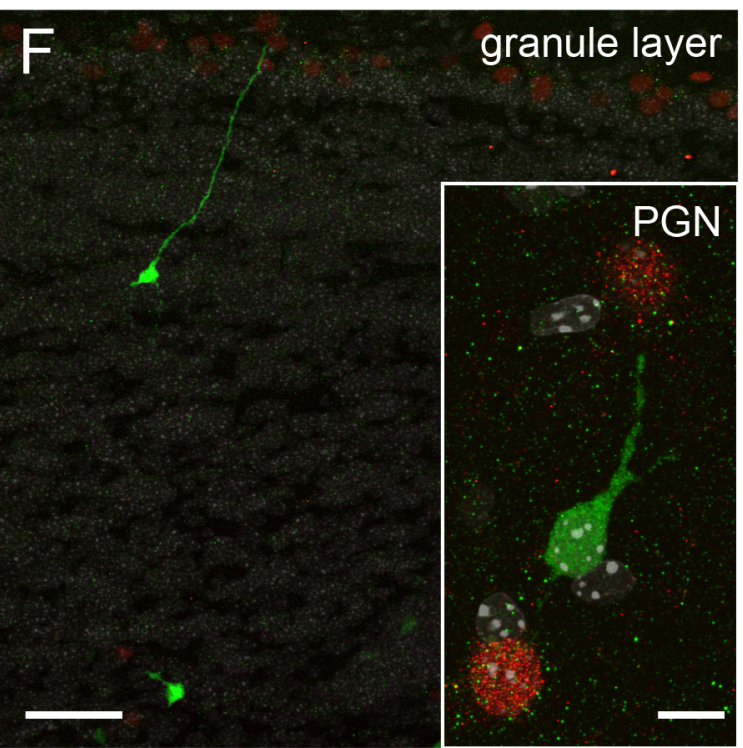

Figure 6 